\title{
A Combinatorial Formula for Kazhdan-Lusztig Polynomials of Sparse Paving Matroids
}

\author{
Kyungyong Lee* \\ Department of Mathematics \\ University of Alabama \\ Alabama, U.S.A. \\ klee94@ua.edu; klee1@kias.re.kr
}

\author{
George D. Nasr ${ }^{\dagger}$ \\ Department of Mathematics \\ University of Oregon \\ Eugene, U.S.A. \\ gdnasr@uoregon.edu
}

\author{
Jamie Radcliffe ${ }^{\ddagger}$ \\ Department of Mathematics \\ University of Nebraska-Lincoln \\ Nebraska, U.S.A \\ jamie.radcliffe@unl.edu
}

Submitted: Jul 27, 2021; Accepted: Nov 29, 2021; Published: Dec 17, 2021

(C) The authors. Released under the CC BY-ND license (International 4.0).

\begin{abstract}
We present a combinatorial formula using skew Young tableaux for the coefficients of Kazhdan-Lusztig polynomials for sparse paving matroids. These matroids are known to be logarithmically almost all matroids, but are conjectured to be almost all matroids. We also show the positivity of these coefficients using our formula. In special cases, such as uniform matroids, our formula has a nice combinatorial interpretation.
\end{abstract}

Mathematics Subject Classifications: 05B35

\section{Introduction}

In 1979, Kazhdan and Lusztig found a polynomial that corresponds to a pair of elements in a Coxeter group [20]. The definition of this polynomial is recursive and uses the

${ }^{*}$ K.L. is partially supported by the University of Alabama, University of Nebraska-Lincoln, Korea Institute for Advanced Study, and the NSF grant DMS-2042786.

${ }^{\dagger}$ G.D.N is supported by the NSF grant DMS-2053243.

${ }^{\ddagger}$ Jamie Radcliffe is supported in part by Simons grant number 429383 . 
Bruhat order to induce a poset structure on the elements of a given Coxeter group. This polynomial, later gets called the Kazhdan-Lusztig polynomial (which we sometimes abbreviate as "KL polynomials"). Since then, the definition of this polynomial have been generalized - for instance, see Stanley's work in [31] and Brenti's continuation of Stanley's work in $[7,8]$ - so that one may define these polynomials using different combinatorial structures. ${ }^{1}$ In 2016, Elias, Proudfoot, and Wakefield did this for matroids [10], which we define here.

Throughout, let $M$ be a matroid, $F$ be a flat of the matroid $M$, rk be the rank function on $M$, and $\chi_{M}$ be the characteristic polynomial for $M$. We denote $M^{F}$ (respectively $M_{F}$ ) for the localization (respectively contraction) for $M$ at $F$. These flats form a poset under inclusion called the lattice of flats, which we denote $L(M)$. The Kazhdan-Lusztig polynomial for $M$, denoted $P_{M}(t)$, is given by the following conditions:

1. If $\operatorname{rk} M=0$, then $P_{M}(t)=1$.

2. If $\operatorname{rk} M>0$, then $\operatorname{deg} P_{M}(t)<\frac{1}{2} \operatorname{rk} M$.

3. $t^{r k M} P_{M}\left(t^{-1}\right)=\sum_{F \in L(M)} \chi_{M^{F}}(t) P_{M_{F}}(t)$.

One familiar with the definition for the KL polynomial of Coxeter groups will notice similarities between it and the definition for KL polynomials of matroids. The primary difference between them is that while the KL polynomial for Coxeter groups is specifically defined for a pair of elements in a Coxeter group, the KL polynomial for matroids is just defined for a matroid. Briefly, this is because any interval in the lattice of flats for a matroid is isomorphic to the lattice of flats of another matroid, but a similar notion is not true for Coxeter groups under the Bruhat order. Hence, one may view KL polynomials for matroids as nicer to work with than those for Coxeter groups.

After their introduction, these polynomials quickly drew active research interest due to their conjectured properties such as the non-negativity of coefficients, and real-rootedness (see $[10,14,15,33])$. These are natural properties to be curious about in light of what we know for the original Kazhdan-Lusztig polynomials. For instance, Elias and Williamson in 2014 [11] proved the non-negativity for all Kazhdan-Lustig polynomials. However, they are not real-rooted, as Polo proved that any polynomial with constant term 1 and nonnegative integer coefficients can be realized as the Kazhdan-Lusztig polynomial for some pair of elements in some Coxeter group [28].

Recently, using algebro-geometric methods, Braden, Huh, Matherne, Proudfoot, and Wang [5] proved the non-negativity of the coefficients for these polynomials. There has also been much effort put into finding relations between these polynomials or generalizations thereof (see $[6,26,32]$ ). These polynomials have been explicitly calculated only for very special classes of matroids (for instance, see [19, 15, 18, 22, 25]), and yet many of the known formulas have left much room for improvement. In particular, as of now, there is no enlightening interpretation for such coefficients. This is also a relevant part

\footnotetext{
${ }^{1}$ The generalized theory is sometimes referred to as the Kazhdan-Lusztig-Stanley theory.
} 
of the history for the original Kahzdan-Lusztig polynomials. For instance, in [2], the authors discuss some combinatorics that led to a non-recursive definition for the original Kazhdan-Lusztig polynomials.

In this paper, we provide a combinatorial formula for the Kazhdan-Lusztig polynomials of sparse paving matroids. We will also provide a proof for the positivity of our formula. While this may not seem necessary in light of [5], we still share our proof as it only utilizes elementary methods.

The class of sparse paving matroids is known to enjoy properties such as being dualclosed and minor-closed. However, what draws research interest to these matroids is a conjecture given by Mayhew, Newman, Welsh, and Whittle [23]. Based on Crapo's and Rota's prediction [9], they conjectured that sparse paving matroids will eventually predominate in any asymptotic enumeration of matroids. That is,

$$
\lim _{n \rightarrow \infty} \frac{s_{n}}{m_{n}}=1
$$

where $s_{n}$ is the number of sparse paving matroids on $n$ elements and $m_{n}$ is the number of matroids on $n$ elements. In pursuit of this conjecture, Pendavingh and van der Pol [24] have shown that

$$
\lim _{n \rightarrow \infty} \frac{\log s_{n}}{\log m_{n}}=1
$$

That is, so far, what we know is that logarithmically almost all matroids are sparse paving matroids.

There are several known characterizations of sparse paving matroids. Let $M$ be a matroid of rank $d$ so that the ground set has $m+d$ elements. Let $\mathcal{B}$ be the set of bases

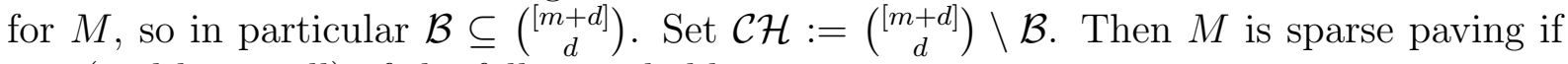
any (and hence all) of the following hold.

1. $\mathcal{C H}$ is the set of circuit-hyperplanes for $M$.

2. For distinct $C, C^{\prime} \in \mathcal{C H}$, we have $\left|C \triangle C^{\prime}\right| \geqslant 4$, where $C \triangle C^{\prime}:=\left(C \backslash C^{\prime}\right) \cup\left(C^{\prime} \backslash C\right)$ is the symmetric difference.

3. Every nonspanning circuit is a hyperplane.

4. $M$ and its dual $M^{*}$ are both paving; that is, their circuits have cardinality at least $d$.

To this end, we let $S_{m, d}(\mathcal{C H})$ be the sparse paving matroid of rank $d$ with ground set $[m+d]$ so that $\mathcal{C H}$ is the set of circuit-hyperplanes.

The last thing we need to define before stating our main result is the object that will allow us to write our combinatorial formula for the coefficients of the polynomials. Define $\operatorname{Skyt}(a, i, b)$ to be the set of fillings of the shape in the figure below so that the rows strictly increase left to right and the columns strictly increase top to bottom with entries in $[a+b+2 i-2]$. We define a related object which we denote $\overline{\operatorname{Skyt}}(i, b)$, the subset of $\operatorname{Skyt}(2, i, b)$ where the value 1 appears at the top of the left-most column. We set 


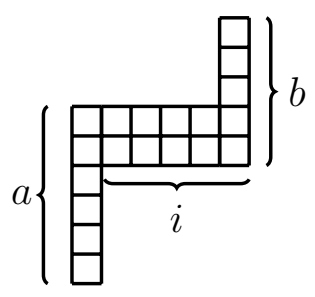

Figure 1: The left-most column has height $a$, followed by $i-1$ columns of height 2 , followed by the right-most column of height $b$.

$\operatorname{skyt}(a, i, b):=\# \operatorname{Skyt}(a, i, b)$ and $\overline{\operatorname{skyt}}(i, b):=\# \overline{\operatorname{Skyt}}(i, b)$. There are some conventions for special values of $a, i$ and $b$, but we leave these for Section 2 .

We are now ready to state our main result.

Theorem 1. Let $c_{m, d}^{i}(\mathcal{C H})$ be the $i$-th coefficient for the Kazhdan-Lusztig polynomial for the sparse paving matroid $S_{m, d}(\mathcal{C H})$. Then

$$
c_{m, d}^{i}(\mathcal{C H})=\operatorname{skyt}(m+1, i, d-2 i+1)-|\mathcal{C H}| \cdot \overline{\operatorname{skyt}}(i, d-2 i+1) .
$$

Moreover, this formula is always non-negative.

What is truly remarkable about this formula is that it is not effected by how the elements of $\mathcal{C H}$ relate to one-another ${ }^{2}$. Keep in mind that $\mathcal{C H}$ could be any set of elements so that their pairwise symmetric difference is at least 4 . Given a fixed $m, d$, and $i$, the value of the coefficient is invariant of selection of $\mathcal{C H}$ so long as $|\mathcal{C H}|$ remains the same. This formula has already inspired other mathematical results since the posting of this paper $[12,13]$.

When $\mathcal{C H}$ is a disjoint collection, we have already shown in [21, Proposition 13] that the formula in Theorem 1 has a manifestly positive interpretation. Consider the subset of $\operatorname{Skyt}(m+1, i, d-2 i+1)$ satisfying at least one of the following three conditions.

- the top entry of the right-most column is 1 ; or

- the bottom entry of the right-most column is greater than $d+|\mathcal{C H}|$; or

- the third entry (from the top) of the left-most column is less than $d+1$.

Then the size of this subset agrees with the formula we give in Theorem 1, by [21, Proposition 13]. In the special case where $\mathcal{C H}=\varnothing$, the second condition becomes tautological as the bottom of the right-most column is guaranteed to be at least $d+1$ for any tableaux. So when $\mathcal{C H}=\varnothing$, we get the entire size of $\operatorname{Skyt}(m+1, i, d-2 i+1)$ as our coefficient, as

\footnotetext{
${ }^{2}$ One may not find this so surprising once they find out the same is true for the characteristic polynomial of sparse paving matroids, as we prove in Corollary 10, but given the definition of Kazhdan-Lusztig polynomials, this fact is not clearly true without Theorem 1 .
} 
Theorem 1 indicates. Also in this case we have $S_{m, d}(\mathcal{C H})=U_{m, d}$, the uniform matroid of rank $d$ on $m+d$ elements. ${ }^{3} 4$

In light of this, we have proven the following conjecture in the case of sparse paving matroids.

Conjecture 2. Let $M$ be a matroid of rank $d$ on $m+d$ elements, and let $c^{i}$ be the $i$-th coefficient for $P_{M}(t)$. Then

$$
c^{i} \leqslant c_{m, d}^{i}(\varnothing) .
$$

That is, among all matroids with rank $d$ and ground set size $m+d$, the Kazhdan-Lusztig polynomial for $U_{m, d}$ has the largest coefficients.

This conjecture was posed by Katie Gedeon. It has no written source, but was communicated to us by Nicholas Proudfoot.

It is also interesting to note that when $\mathcal{C H}$ is a disjoint collection, $S_{m, d}(\mathcal{C H})$ can be seen to be representable. This in turn gives a combinatorial formula for the intersection cohomology Poincaré polynomial of the corresponding reciprocal plane over a finite field, thanks to [10]. In general, though, almost all sparse paving matroids are not representable. This is due in large part to Nelson [29] who showed that asymptotically almost all matroids are not representable. In particular, his work implies that the logarithmic growth of representable matroids is bounded by a polynomial. Meanwhile, the logarithmic growth of matroids in general are known to have at least exponential growth, and so the same must be true for sparse paving matroids.

One final thing to note that is interesting about our formula is that if $m+1=2$ or $d-2 i+1=2$, then $\operatorname{skyt}(m+1, i, d-2 i+1)$ becomes equal to a well-known number, namely the number of polygon dissections [30]. Hence, when $m+1=d-2 i+1=2$, it becomes a Catalan number. ${ }^{5}$

This paper proceeds as follows. In section 2, we further discuss the elements of $\operatorname{Skyt}(a, i, b)$ and $\overline{\operatorname{Skyt}}(i, b)$. We also bring up some important conventions and useful identities for $\operatorname{skyt}(a, i, b)$ and $\overline{\operatorname{skyt}}(a, i, b)$. In section 3 , we discuss flats, localizations, contractions, and characteristic polynomial for $S_{m, d}(\mathcal{C H})$. In section 4 , we verify the formula for the Kazhdan-Lusztig polynomial of $S_{m, d}(\mathcal{C H})$ given in Theorem 1. We then give some useful upper bounds on $|\mathcal{C H}|$ in section 5 . We use these bounds to prove the non-negative part of Theorem 1, which we do in section 6 . We end the paper with some integral identities we use throughout the paper in section 7 .

\footnotetext{
${ }^{3}$ The first (and only known) manifestly positive integral interpretation for uniform matroids was given in $[17$, Remark 3.4$]$, which requires possibly many Young diagrams.

${ }^{4}$ Those familiar with matroid theory may be more used to the notation $U_{d, n}$ to the uniform matroid of rank $d$ on $n$ elements. We chose our notation to be consistent with other authors publishing results for Kazhdan-Lusztig polynomials for uniform matroids.

${ }^{5}$ This connection to polygon dissections was already mentioned in several places, namely in Remark 1.3 in [27] and Remark 5.3 of [18], but with the discovery of our combinatorial object, this fact follows directly from [30].
} 


\section{Skew Young Tableaux}

Consider the following shape. A legal filling of the above shape involves placing each

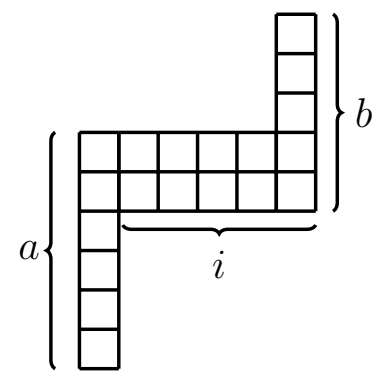

Figure 2: The left-most column has height $a$, followed by $i-1$ columns of height 2 , followed by the right-most column of height $b$.

number from $\{1,2, \ldots, a+2 i+b-2\}$ into the squares such that the values in the columns and rows strictly increase going down and right, respectively. Note that this is the same restriction on the entries of a standard Young tableau, but the above shape does not fit the description of the typical Young tableau. We refer to a legal filling of the above shape as a skew young tableau, and denote $\operatorname{Skyt}(a, i, b)$ as the set of such legal fillings, and denote $\operatorname{skyt}(a, i, b):=\# \operatorname{Skyt}(a, i, b)$.

For our tableaux to be defined, we need $a, b \geqslant 2$ and $i \geqslant 1$, but our formula in Theorem 1 may be used for other non-negative values of $a, b$, and $i$. Hence, there are some conventions we have set for the few exceptional values that can occur so that our formula still works.

- If $i=0$, then $\operatorname{skyt}(a, i, b)=1$.

- If $i>0$ and at least one of $a$ or $b$ is less than 2 , then $\operatorname{skyt}(a, i, b)=0$.

We also define a related collection of objects, which we denote $\overline{\operatorname{Skyt}}(i, b)$. This set is the subset of $\operatorname{Skyt}(2, i, b)$ so that 1 is always the entry at the top of the left-most column. The size of $\overline{\operatorname{Skyt}}(i, b)$ is denoted $\overline{\operatorname{skyt}}(i, b)$. By convention, $\overline{\operatorname{skyt}}(i, b)=0$ if $i=0$.

In [21, Lemma 4], we proved the following result.

Lemma 3. For $a, b \geqslant 2$ and $i \geqslant 1$, we have

$$
\operatorname{skyt}(a, i, b)=\frac{1}{i !(a-2) !(a+i-1)} \sum_{k=0}^{b-2}(-1)^{k}\left(\begin{array}{c}
a+b+2 i-2 \\
b-2-k
\end{array}\right) \frac{(a+2 i+k) !(k+1)}{(a+i+k)(i+k+1) !}
$$

This formula for $\operatorname{skyt}(a, i, b)$ is useful in proving the following.

Lemma 4. For $b \geqslant 2$ and $i \geqslant 1$, we have

$$
\overline{\operatorname{skyt}}(i, b)=\frac{1}{(i+1) !} \sum_{k=0}^{b-2}(-1)^{k}\left(\begin{array}{c}
b+2 i-1 \\
b-2-k
\end{array}\right) \frac{(2 i+k+2) !(k+1)}{(i+k+2) !} .
$$


Proof. Observe that $\overline{\operatorname{Skyt}}(i, b) \subseteq \operatorname{Skyt}(2, i, b)$. In particular, note that the tableaux in $T:=\operatorname{Skyt}(2, i, b) \backslash \overline{\operatorname{Skyt}}(i, b)$ are those where the value 1 appears at the top of the rightmost column. One can achieve a bijection between $\operatorname{Skyt}(2, i, b-1)$ and $T$ : For any tableaux $\alpha$ in $\operatorname{Skyt}(2, i, b-1)$, increase each numerical value in $\alpha$ by 1 , and then extend the rightmost column by adding one cell at the top of the column, placing the number 1 in this position. Hence,

$$
\begin{aligned}
\overline{\operatorname{skyt}}(i, b) & =\operatorname{skyt}(2, i, b)-\operatorname{skyt}(2, i, b-1) \\
& =\frac{1}{(i+1) !} \sum_{k=0}^{b-2}(-1)^{k}\left(\left(\begin{array}{c}
b+2 i \\
b-2-k
\end{array}\right)-\left(\begin{array}{c}
b-1+2 i \\
b-3-k
\end{array}\right)\right) \frac{(2 i+k+2) !(k+1)}{(i+k+2) !} \\
& =\frac{1}{(i+1) !} \sum_{k=0}^{b-2}(-1)^{k}\left(\begin{array}{c}
b+2 i-1 \\
b-2-k
\end{array}\right) \frac{(2 i+k+2) !(k+1)}{(i+k+2) !},
\end{aligned}
$$

by Pascal's identity.

One can achieve two formulas for $\operatorname{skyt}(a, i, b)$ and $\overline{\operatorname{skyt}}(i, b)$ that avoid alternating sums. We will need a few integral identities to produce these formulas. These identities can be found in section 7 , but are referenced as they are needed in the proofs that follow. Throughout, $(x)^{(n)}$ is the rising factorial $(x)(x+1) \cdots(x+n-1)$ for integers $x$ and $n$.

We start with the formula for $\operatorname{skyt}(a, i, b)$.

Lemma 5. For $a, b \geqslant 2$ and $i \geqslant 1$, we have

$$
\operatorname{skyt}(a, i, b)=\left(\begin{array}{c}
a+i-2 \\
i
\end{array}\right)\left(\begin{array}{c}
a+b+2 i-2 \\
b+i-1
\end{array}\right) \sum_{k=0}^{b-2} \frac{\left(\begin{array}{c}
b+i-k-3 \\
i-1
\end{array}\right)}{\left(\begin{array}{c}
a+i+k \\
k+1
\end{array}\right)} .
$$

Proof. One can rewrite Lemma 3 as

$$
\operatorname{skyt}(a, i, b)=\frac{(a+b+2 i-2) !}{i !(a-2) !(a+i-1)(b-2) !} \sum_{k=0}^{b-2}(-1)^{k}\left(\begin{array}{c}
b-2 \\
k
\end{array}\right) \frac{1}{(a+i+k)(k+2)^{(i)}} .
$$
Let

We can recover this sum for $\operatorname{skyt}(a, i, b)$ by applications of integrals to a polynomial.

$$
f(x, y)=\frac{(a+b+2 i-2) ! x y^{a+i-1}(1-x y)^{b-2}}{i !(a-2) !(a+i-1)(b-2) !} .
$$

Our integrals are broken up into three parts.

(a) First find $g(x)$, where $g(x):=\int_{0}^{1} f(x, y) d y$; then

(b) find $h_{i-1}\left(x_{i-1}\right):=\int_{0}^{x_{i-1}} h_{i-2}\left(x_{i-2}\right) d x_{i-2}$, where $h_{1}\left(x_{1}\right):=\int_{0}^{x_{1}} g\left(x_{0}\right) d x_{0}$ and $x_{0}, x_{1}, \ldots, x_{i-1}$ are $i$ variables; then 
(c) solve $\int_{0}^{1} h_{i-1}\left(x_{i-1}\right) d x_{i-1}$.

It is not difficult to show that, if $(1-x y)^{b-2}$ is written using the binomial expansion, part (c) will give the equation for $\operatorname{skyt}(a, i, b)$ found in equation (1) above. To get the statement of Lemma 5, we apply these three steps to $f(x, y)$ directly as written.

First, we use Corollary 27 to do part (a).

$$
g(x):=\int_{0}^{1} f(x, y) d y=\frac{(a+b+2 i-2) !(a+i-1) !}{i !(a-2) !(a+i-1)} \sum_{k=0}^{b-2} \frac{(1-x)^{b-k-2} x^{k+1}}{(a+i+k) !(b-k-2) !} .
$$

To complete parts (b) and (c) we apply Proposition 29 to get

$$
\int_{0}^{1} h_{i-1}\left(x_{i-1}\right) d x_{i-1}=\frac{(a+b+2 i-2) !(a+i-1) !}{i !(a-2) !(a+i-1)(i-1) !(b+i-1) !} \sum_{k=0}^{b-2} \frac{(b+i-k-3) !(k+1) !}{(a+i+k) !(b-k-2) !}
$$

This gets us a manifestly positive sum, and all that is left to get our desired result is to perform some algebraic manipulations. One can combine the terms $(b+i-k-3)$ !, $(b-k-2)$ !, and $(i-1)$ ! to give $\left(\begin{array}{c}b+i-k-3 \\ i-1\end{array}\right)$. Then combine $(a+i-1) !,(k+1) !$, and $(a+i+k)$ ! to get $\left(\begin{array}{c}a+i+k \\ k+1\end{array}\right)$. Then scaling by $\frac{(a+i-2) !}{(a+i-2) !}$ allows us to group the remaining factors into binomial coefficients giving

$$
\left(\begin{array}{c}
a+i-2 \\
i
\end{array}\right)\left(\begin{array}{c}
a+b+2 i-2 \\
b+i-1
\end{array}\right) \sum_{k=0}^{b-2} \frac{\left(\begin{array}{c}
b+i-k-3 \\
i-1
\end{array}\right)}{\left(\begin{array}{c}
a+i+k \\
k+1
\end{array}\right)}
$$

Remark 6.

1. While having a manifestly positive formula for $\operatorname{skyt}(a, i, b)$ is nice, it is unfortunate that, in general, the terms of the sum in Lemma 5 are not necessarily integers, even if you scale them by $\left(\begin{array}{c}a+i-2 \\ i\end{array}\right)$ and $\left(\begin{array}{c}a+b+2 i-2 \\ b+i-1\end{array}\right)$.

2. It will be useful to rewrite Lemma 5 using a common denominator. We can do this by rewriting the binomials in the sum using the falling factorial $(x)_{(n)}:=x(x-$ 1) $\cdots(x-n+1)$. Rewriting the sum gives

$$
\begin{aligned}
& \sum_{k=0}^{b-2} \frac{\left(\begin{array}{c}
b+i-k-3 \\
i-1
\end{array}\right)}{\left(\begin{array}{c}
a+i+k \\
k+1
\end{array}\right)} \\
& =\sum_{k=0}^{b-2} \frac{(b+i-k-3)_{b-k-2}(k+1) !}{(b-k-2) !(a+i+k)_{(k+1)}} \\
& =\frac{\sum_{k=0}^{b-2}(b+i-k-3)_{b-k-2}(k+1) !(b-2)_{(k)}(a+i+b-2)_{(b-k-2)}}{(b-2) !(a+i+b-2)_{(b-1)}}
\end{aligned}
$$


We will find this version useful later, though it is not as concise as the original formula.

Using similar methods, we can find a formula for $\overline{\operatorname{skyt}}(i, b)$ which not only avoids an alternating sum, but is in fact a single term.

Lemma 7. For $a, b \geqslant 2$ and $i \geqslant 1$, we have

$$
\overline{\operatorname{skyt}}(i, b)=\frac{2(b+2 i-1) !}{(i+1) !(i-1) !(b-2) !(b+i)(b+i-2)} .
$$

Proof. One can rewrite Lemma 7 as

$$
\overline{\operatorname{skyt}}(i, b)=\frac{(b+2 i-1) !}{(i+1) !(b-2) !} \sum_{k=0}^{b-2}(-1)^{k}\left(\begin{array}{c}
b-2 \\
k
\end{array}\right) \frac{(2 i+k+2)}{(k+2)^{(i+1)}} .
$$

We can recover this sum for $\overline{\operatorname{skyt}}(a, i, b)$ by applications of a derivative and integrals to a polynomial. Let

$$
f(x, y)=\frac{(b+2 i-1) ! x y^{2 i+2}(1-x y)^{b-2}}{(i+1) !(b-2) !} .
$$

We break up our plan for applications of a derivative and integrals into three parts.

(a) First solve $g(x):=\left.\frac{d}{d y} f(x, y)\right|_{y=1}$; then

(b) find $h_{i}\left(x_{i}\right):=\int_{0}^{x_{i}} h_{i-1}\left(x_{i-1}\right) d x_{i-1}$, where $h_{1}\left(x_{1}\right):=\int_{0}^{x_{1}} g\left(x_{0}\right) d x_{0}$ and $x_{0}, x_{1}, \ldots, x_{i}$ are $i+1$ variables; then finally

(c) find $\int_{0}^{1} h_{i}\left(x_{i}\right) d x_{i}$.

If one writes $(1-x y)^{b-2}$ using the binomial expansion, part (c) outputs the equation for skyt found in equation (2) above. We claim that leaving $f(x, y)$ as written and then applying these three steps lead to the statement of Lemma 7.

First, for part (a), we observe that

$$
g(x)=\left.\frac{d}{d y} f(x, y)\right|_{y=1}=\frac{2(i+1)(b+2 i-1) !}{(i+1) !(b-2) !} x(1-x)^{b-2}-\frac{(b-2)(b+2 i-1) !}{(i+1) !(b-2) !} x^{2}(1-x)^{b-3} .
$$

We do parts (b) and (c) simultaneously due to Proposition 29. This gives

$$
\begin{aligned}
\int_{0}^{1} h_{i}\left(x_{i}\right) d x_{i} & =\frac{2(i+1)(b+2 i-1) !}{(i+1) !(b-2) !} \frac{(b-2+i) !}{i !(b+i) !}-\frac{(b-2)(b+2 i-1) !}{(i+1) !(b-2) !} \frac{2(b-3+i) !}{i !(b+i) !} \\
& =\frac{2(b+2 i-1) !}{(i+1) !(i-1) !(b-2) !(b+i)(b+i-2)} .
\end{aligned}
$$




\section{Flats, Contractions, Localizations, and Characteristic Poly- nomials for $S_{m, d}(\mathcal{C H})$}

Throughout, let $F$ be a flat, that is, a set which is maximal with respect to its rank. For a matroid $M$, recall that $M^{F}$ (respectively, $M_{F}$ ) denotes the localization (respectively, contraction) of $M$ at $F$. By $M^{F}$, we mean the matroid with ground set $F$, whose independent sets are those subsets of $F$ that are also independent in $M$. By $M_{F}$, we mean the matroid with ground set $M \backslash F$, whose independent sets are those subsets whose union with a basis for $F$ is independent in $M$.

First, we discuss the flats of $S_{m, d}(\mathcal{C H})$. It is an elementary exercise to verify the following.

Proposition 8. The flats of $S_{m, d}(\mathcal{C H})$ are composed of

1. the sets of cardinality at most $d-2$;

2. the sets of cardinality $d-1$ not contained in any element of $\mathcal{C H}$;

3. the elements of $\mathcal{C H}$;

4. $[m+d]$.

With this, we can now discuss the localizations and contractions of $S_{m, d}(\mathcal{C H})$. First, recall the localizations and contractions of $U_{m, d}$, the uniform matroid of rank $d$ with ground set $[m+d]$, which are given by

$$
\left(U_{m, d}\right)^{F}= \begin{cases}U_{m, d} & F=[m+d] \\ U_{0,|F|} & F \neq[m+d]\end{cases}
$$

and

$$
\left(U_{m, d}\right)_{F}=\left\{\begin{array}{ll}
U_{0,0} & F=[m+d] \\
U_{m, d-|F|} & F \neq[m+d]
\end{array} .\right.
$$

The corresponding equations for $S_{m, d}(\mathcal{C H})$ can also be described in a similar manner. In what follows, if $F$ is a flat, then we define $\mathcal{C H}(F):=\{C \backslash F: C \in \mathcal{C H}$ such that $F \subseteq$ $C$. It is worth noting that if $\mathcal{C H}$ is the set of circuit-hyperplanes for a sparse paving matroid, then so is $\mathcal{C H}(F)$, so long as $F$ is strictly contained in some circuit-hyperplane. One way to check this is by verifying $\mathcal{C H}(F)$ satisfies the condition that any pair has symmetric difference at least 4 .

\section{Proposition 9.}

$$
S_{m, d}(\mathcal{C H})^{F}= \begin{cases}S_{m, d}(\mathcal{C H}) & F=[m+d] \\ U_{1, d-1} & F \in \mathcal{C H} \\ U_{0,|F|} & \text { otherwise }\end{cases}
$$


and

$$
S_{m, d}(\mathcal{C H})_{F}=\left\{\begin{array}{ll}
S_{m, d}(\mathcal{C H}) & F=\varnothing \\
U_{m-1,1} & F \in \mathcal{C H} \\
S_{m, d-|F|}(\mathcal{C H}(F)) & \varnothing \subsetneq F \subsetneq C, \text { for some } C \in \mathcal{C H} \\
\left(U_{m, d}\right)_{F} & \text { otherwise }
\end{array} .\right.
$$

Proof. For the localization, the only new case necessary to mention in comparison to the uniform case is for $F \in \mathcal{C H}$; the other cases follow from the uniform case. The localization of this matroid at $F$ treats $F$ as the ground set, with independent sets being those that are independent in $S_{m, d}(\mathcal{C H})$. We know every proper subset of $F$ is independent, giving $U_{1, d-1}$.

Now for the contraction. If we have $F \nsubseteq C$ for all $C \in \mathcal{C H}$, then the structure of $S_{m, d}(\mathcal{C H})_{F}$ is exactly that of $\left(U_{m, d}\right)_{F}$. For the case where $F \in \mathcal{C H}$, we want the subsets of $S:=[m+d] \backslash F$ such that their union with a basis for $F$ is independent in $S_{m, d}(\mathcal{C H})$. The bases for $F$ are the elements of $\left(\begin{array}{c}F \\ d-1\end{array}\right)$. Note if $B \in\left(\begin{array}{c}{[m+d]} \\ d\end{array}\right)$ satisfies $|B \triangle F|=2$, then $B$ is independent in $S_{m, d}(\mathcal{C H})$. This means the desired subsets of $S$ are the empty set and every singleton of $S$. This gives a matroid isomorphic to $U_{m-1,1}$. Finally, when $\varnothing \subsetneq F \subsetneq C$, for some $C \in \mathcal{C H}$, note that $F$ is independent, and hence a basis for itself. Thus, the independent sets for $S_{m, d}(\mathcal{C H})_{F}$ are the subsets $X$ of $[m+d] \backslash F$ so that $X \cup F$ is independent in $S_{m, d}(\mathcal{C H})$. That is, $|X| \leqslant d-|F|$. When $|X|<d-|F|,|X \cup F|<d$ and every subset of $[m+d]$ of size smaller than $d$ is independent. When $|X|=d-|F|$, $X \cup F$ is a basis for $S_{m, d}(\mathcal{C H})$ if and only if $X \cup F \neq C$, for any $C \in \mathcal{C H}$, which is true if and only if $X \notin \mathcal{C H}(F)$. That is, we get a matroid isomorphic to $S_{m, d-|F|}(\mathcal{C H}(F))$.

With these in mind, we can now compute the characteristic equation for all localizations for $S_{m, d}(\mathcal{C H})$. However, by Proposition 9, we equivalently just need to find the characteristic polynomial for $U_{m, d}$ and $S_{m, d}(\mathcal{C H})$.

First, recall that for a (loopless) matroid $M$, the characteristic polynomial is given by

$$
\chi_{M}(t)=\sum_{F \in L(M)} \mu_{L(M)}(\hat{\mathbf{0}}, F) t^{\mathrm{rk} M-\mathrm{rk} F},
$$

where $L(M)$ is the lattice of flats for matroid $M$. For uniform matroids, we have

$$
\chi_{U_{m, d}}(t)=(-1)^{d}\left(\begin{array}{c}
m+d-1 \\
d-1
\end{array}\right)+\sum_{i=0}^{d-1}(-1)^{i}\left(\begin{array}{c}
m+d \\
i
\end{array}\right) t^{d-i} .
$$

Knowing this simplifies our computation of $\chi_{S_{m, d}}(\mathcal{C H})$.

Corollary 10. Let $c=|\mathcal{C H}|$. Then

$$
\begin{aligned}
\chi_{S_{m, d}(\mathcal{C H})}(t) & =(-1)^{d}\left(\begin{array}{c}
m+d-1 \\
d-1
\end{array}\right)-(-1)^{d} c \\
& +t(-1)^{d-1}\left(\left(\begin{array}{c}
m+d \\
d-1
\end{array}\right)-c\right)+\sum_{i=0}^{d-2}(-1)^{i}\left(\begin{array}{c}
m+d \\
i
\end{array}\right) t^{d-i} .
\end{aligned}
$$


It is noteworthy that this characteristic polynomial is the same for all choices of $\mathcal{C H}$ that have the same size.

Proof of Corollary 10. One can see this by combining Proposition 8 with the fact that the characteristic function of a matroid $M$ may be rewritten as

$$
\chi_{M}(t)=\sum_{S \subseteq E}(-1)^{|S|} t^{\mathrm{rk}(M)-\mathrm{rk}(S)},
$$

where $E$ is the groundset of $M$.

It will be helpful to restate this proposition in the following way for when we prove Theorem 1.

Corollary 11. (Corollary 10 restated.)

$$
\left[t^{i}\right] \chi_{S_{m, d}(\mathcal{C H})}=\left\{\begin{array}{ll}
(-1)^{d}\left(\begin{array}{c}
m+d-1 \\
d-1
\end{array}\right)-c(-1)^{d} & i=0 \\
(-1)^{d-1}\left(\begin{array}{c}
m+d \\
d-1
\end{array}\right)-c(-1)^{d-1} & i=1 \\
(-1)^{d-i}\left(\begin{array}{c}
m+d \\
d-i
\end{array}\right) & 2 \leqslant i \leqslant d
\end{array} .\right.
$$

\section{Kazhdan-Lusztig Polynomials for Sparse Paving Matroids}

This section is dedicated to justifying the combinatorial formula given in Theorem 1 . We restate this part here for convenience, as its own Theorem.

Theorem 12. Let $c_{m, d}^{i}(\mathcal{C H})$ be the $i$-th coefficient for the Kazhdan-Lusztig polynomial for the sparse paving matroid $S_{m, d}(\mathcal{C H})$. Then

$$
c_{m, d}^{i}(\mathcal{C H})=\operatorname{skyt}(m+1, i, d-2 i+1)-|\mathcal{C H}| \cdot \overline{\operatorname{skyt}}(i, d-2 i+1) .
$$

Remark 13. For some values of $m, d$, and $i$, we need to use our conventions set in place for $\operatorname{skyt}(a, i, b)$ and $\overline{\operatorname{skyt}}(i, b)$ in section 2 for our formula to truly work.

- $[10$, Proposition 2.11] shows that the degree 0 term always has coefficient 1 . That is, when $i=0$, our formula must always return 1 .

- When $d=0$ we are forced to have $P_{S_{m, d}(\mathcal{C H})}(t)=1$.

- When $0<d<3$, the degree requirement on Kazhdan-Lusztig polynomials forces $P_{S_{m, d}(\mathcal{C H})}(t)$ to have degree 0 . Namely, in this case, we have $P_{S_{m, d}(\mathcal{C H})}(t)=1$, again by [10, Proposition 2.11].

- When $m=0$, note that $\mathcal{C H}$ is forced to be empty and $S_{m, d}(\mathcal{C H})$ becomes $U_{0, d}$. It is shown in [10, Proposition 2.7] that $P_{M_{1} \oplus M_{2}}(t)=P_{M_{1}}(t) P_{M_{2}}(t)$ for matroids $M_{1}$ and $M_{2}$. With this, one can verify that $P_{U_{0, d}}(t)=1$ by seeing that $P_{U_{0,1}}(t)=1$ based on the $d<3$ discussion above. 
In all cases, our conventions guarantee we get the right values. Besides these cases, our conventions are not needed for our formula, and we are guaranteed that $S_{m, d}(\mathcal{C H})$ has more interesting structure than that of the boolean lattice.

The following technical result will be crucial in demonstrating why the formula given in Theorem 12 only depends on $|\mathcal{C H}|$, and not the relationship between the elements of $\mathcal{C H}$.

Lemma 14. Let $c, i \in \mathbb{N} \cup\{0\}$. For $I \subseteq[c]$, let $x_{I}$ be a variable. Let $g(k)$ and $h(k)$ are functions varying in $k$. Then

$$
-\sum_{\substack{J \subseteq[c] \\|J| \geqslant 2}}(-1)^{|J|} x_{J} \sum_{k=0}^{i} g(k)=\sum_{\varnothing \subsetneq I \subseteq[c]} \sum_{\substack{\subseteq \subseteq J \subseteq[c] \\|J| \geqslant 2}}(-1)^{|J|-|I|} x_{J} \sum_{k=0}^{i}(g(k)-|I| h(k)) .
$$

Proof. We show that the term with $x_{J}$ on both sides of the statement of the lemma is the same for every $J \subseteq[c]$, where $|J| \geqslant 2$. We start with the coefficient of $x_{J}$ on the right side. We note that the terms with $x_{J}$ appear for each $I$ that is contained in $J$, where $|I| \geqslant 1$. Hence, the term with $x_{J}$ on the right hand side of the statement of the Lemma is

$$
\begin{aligned}
& \sum_{\ell=1}^{|J|}(-1)^{|J|-\ell} x_{J}\left(\begin{array}{c}
|J| \\
\ell
\end{array}\right) \sum_{k=0}^{i}(g(k)-\ell h(k)) \\
& =x_{J}(-1)^{|J|} \sum_{\ell=1}^{|J|}\left(\begin{array}{c}
|J| \\
\ell
\end{array}\right)(-1)^{\ell} \sum_{k=0}^{i}(g(k)-\ell h(k)) \\
& =x_{J}(-1)^{|J|}\left(\sum_{k=0}^{i} g(k) \sum_{\ell=1}^{|J|}(-1)^{\ell}\left(\begin{array}{c}
|J| \\
\ell
\end{array}\right)-\sum_{k=0}^{i} h(k) \sum_{\ell=1}^{|J|}(-1)^{\ell} \ell\left(\begin{array}{c}
|J| \\
\ell
\end{array}\right)\right) \\
& =x_{J}(-1)^{|J|}\left(-\sum_{k=0}^{i} g(k)\right),
\end{aligned}
$$

since we know in general we have the identities $\sum_{\ell=0}^{n}(-1)^{\ell}\left(\begin{array}{l}n \\ \ell\end{array}\right)=0$ for $n \geqslant 1$ and $\sum_{\ell=0}^{n}(-1)^{\ell}\left(\begin{array}{l}n \\ \ell\end{array}\right) \ell=0$ for $n \geqslant 2$. Note that there is exactly one time where $x_{J}$ appears exactly once, and the corresponding term is $-x_{J}(-1)^{|J|} \sum_{k=0}^{i} g(k)$.

We now prove the desired formula for $c_{m, d}^{i}(\mathcal{C H})$.

Proof of Theorem 12. Let $M:=S_{m, d}(\mathcal{C H})$, and set $c:=|\mathcal{C H}|$. Recall that the definition for the Kazhdan-Lusztig polynomial is that it satisfies the following recurrence,

$$
t^{r k M} P_{M}\left(t^{-1}\right)=\sum_{F \text { a flat }} \chi_{M^{F}}(t) P_{M_{F}}(t),
$$


which may be rewritten as

$$
t^{r k M} P_{M}\left(t^{-1}\right)-P_{M}(t)=\sum_{F \text { a non-empty flat }} \chi_{M^{F}}(t) P_{M_{F}}(t)
$$

Recall that $\operatorname{deg} P_{M}(t)<\frac{1}{2} d$, and so the power of each monomial in $t^{d} P_{M}\left(t^{-1}\right)$ is strictly larger than $\frac{1}{2} d$. Hence, our goal is to show that for $0 \leqslant i<\frac{1}{2} d$ we have

$$
-\operatorname{skyt}(m+1, i, d-2 i+1)+c \cdot \overline{\operatorname{skyt}}(i, d-2 i+1)=\left[t^{i}\right] \sum_{F \text { a non-empty flat }} \chi_{M^{F}}(t) P_{M_{F}}(t) .
$$

Using our work from Proposition 9, and consolidating common factors involving the various flats in $\mathcal{C H}$, we can rewrite the right of equation (3) to be

$$
+\left[t^{i}\right] \chi_{S_{m, d}(\mathcal{C H})}+c\left[t^{i}\right] \chi_{U_{1, d-1}} P_{U_{m-1,1}}\left[\sum_{\substack{\varnothing \subsetneq F \subsetneq C \\ \text { For some } C \in \mathcal{C H}}}\left[t^{i}\right] \chi_{U_{0,|F|} \mid} P_{S_{m, d-|F|}(\mathcal{C H}(F))}+\sum_{\substack{\varnothing \subsetneq F \subsetneq[m+d] \\ F \nsubseteq C \forall C \in \mathcal{C H}}}\left[t^{i}\right] \chi_{U_{0,|F|} \mid} P_{U_{m, d-|F|}}\right.
$$

where the first term corresponds to the case where $F=[m+d]$, and the second where $F \in \mathcal{C H}$.

By Proposition 11, we are required to break this up into three case: $i=0, i=1$, and $2 \leqslant i<d / 2$ if we are to write this out explicitly. Note we can write everything explicitly except $P_{S_{m, d-|F|}(\mathcal{C H}(F))}$. Hence, we proceed by induction on the matroid rank $d$, noting that $d>d-|F|$ since for the corresponding summand $F$ is never empty.

We now define some notation in order to rewrite the summations appearing in (4). Let $I \subseteq[c]$ and $C_{i} \in \mathcal{C H}$. We define $C_{I}:=\bigcap_{i \in I} C_{i}$ and denote $c_{I}:=\left|C_{I}\right|$. By convention, $C_{\varnothing}=[m+d]$. Recall that $\mathcal{C H}(F):=\{C \backslash F: C \in \mathcal{C H}$ such that $F \subseteq C\}$. Let $j$ be an integer and define the following sum indexed by $J$ :

$$
\Phi_{j}(I):=\sum_{I \subseteq J \subseteq[c]}(-1)^{|J|-|I|}\left(\begin{array}{c}
c_{J} \\
j
\end{array}\right) .
$$

If $j$ is selected appropriately, $\Phi_{j}(I)$ counts the number of flats of rank $j$ contained in $C_{I}$, but not in any $C_{J}$ so that $C_{J} \subseteq C_{I}$. Hence, $F$ is a flat counted by $\Phi_{j}(I)$ if and only if $\mathcal{C H}(F)=\left\{C_{i} \backslash F: i \in I\right\}$. What we will infer from this is that $|\mathcal{C H}(F)|=|I|$.

We can now rewrite equation (4). We use the Kronecker delta function $\delta(i, j)=\left\{\begin{array}{ll}1 & i=j \\ 0 & i \neq j\end{array}\right.$ to combine the cases for $i=1$ and $2 \leqslant i<d / 2$. 
$i=0$ :

$$
\begin{aligned}
& (-1)^{d}\left(\begin{array}{c}
m+d-1 \\
d-1
\end{array}\right)-c(-1)^{d}+c(-1)^{d-1}\left(\begin{array}{l}
d-1 \\
d-2
\end{array}\right) \\
& +\sum_{j=1}^{d-2} \sum_{\varnothing \subsetneq I \subseteq[c]} \Phi_{j}(I)(-1)^{j}(\operatorname{skyt}(m+1,0, d-j+1)-|I| \cdot \overline{\operatorname{skyt}}(0, d-j+1)) \\
& +\sum_{j=1}^{d-1} \Phi_{j}(\varnothing)(-1)^{j} \operatorname{skyt}(m+1,0, d-j+1) .
\end{aligned}
$$

$i>0$ :

$$
\begin{aligned}
& (-1)^{d-i}\left(\begin{array}{c}
m+d-1 \\
d-i
\end{array}\right)-c(-1)^{d-1} \delta(i, 1)+c(-1)^{d-1-i}\left(\begin{array}{c}
d \\
d-1-i
\end{array}\right) \\
& +\sum_{j=1}^{d-2} \sum_{\varnothing \subsetneq I \subseteq[c]} \Phi_{j}(I) \sum_{k=0}^{i}(-1)^{j-i+k}\left(\begin{array}{c}
j \\
j-i+k
\end{array}\right)(\operatorname{skyt}(m+1, k, d-j-2 k+1) \\
& -|I| \overline{\operatorname{skyt}}(k, d-j-2 k+1)) \\
& +\sum_{j=1}^{d-1} \Phi_{j}(\varnothing) \sum_{k=0}^{i}(-1)^{j-i+k}\left(\begin{array}{c}
j \\
j-i+k
\end{array}\right) \operatorname{skyt}(m+1, k, d-j-2 k+1) .
\end{aligned}
$$

In both cases, the sum running from $j=1$ to $j=d-2$ is the summand in equation (4) over $\varnothing \subsetneq F \subsetneq C$ for $C \in \mathcal{C H}$, since the flats contained in $C$ have size at most $d-2$. The other sum running from $j=1$ to $j=d-1$ corresponds to the summand in equation (4) over $\varnothing \subsetneq F \subsetneq[m+d]$ such that $F \nsubseteq C$ for all $C \in \mathcal{C H}$.

To simplify things further, first, note that

$$
\Phi_{d-1}(\varnothing)=\left(\begin{array}{c}
m+d \\
d-1
\end{array}\right)-c\left(\begin{array}{c}
d \\
d-1
\end{array}\right)
$$

By construction, $\Phi_{d-1}(\varnothing)$ counts the rank $d-1$ flats contained in no element of $\mathcal{C H}$. Recall that the only rank $d-1$ flats are those not contained in any circuit-hyperplane.

Next, note that many terms from the two sums running over $j$ in both the $i=0$ and $i>0$ case will cancel as a result of Lemma 14. Fix $j \leqslant d-2$ and suppose $J \subseteq[c]$. Set

- $x_{J}:=\left(\begin{array}{c}c_{J} \\ j\end{array}\right)$,

- $g(k):=(-1)^{j-i+k}\left(\begin{array}{c}j \\ j-i+k\end{array}\right) \operatorname{skyt}(m+1, k, d-j-2 k+1)$, and

- $h(k):=(-1)^{j-i+k}\left(\begin{array}{c}j \\ j-i+k\end{array}\right) \overline{\operatorname{skyt}}(k, d-j-2 k+1)$.

This allows us to rewrite our two cases in the following way. 
$i=0$ :

$$
\begin{aligned}
& (-1)^{d}\left(\begin{array}{c}
m+d-1 \\
d-1
\end{array}\right)-c(-1)^{d}+c(-1)^{d-1}\left(\begin{array}{l}
d-1 \\
d-2
\end{array}\right) \\
& +\sum_{j=1}^{d-2} \sum_{\varnothing \subseteq I \subseteq[c]} \sum_{I \subseteq J \subseteq[c]}(-1)^{|J|-|I|} x_{J}(g(0)-|I| h(0)) \\
& +\sum_{j=1}^{d-1} \sum_{\varnothing \subseteq J \subseteq[c]}(-1)^{|J|} x_{J} g(0) .
\end{aligned}
$$

$i>0$ :

$$
\begin{aligned}
& (-1)^{d-i}\left(\begin{array}{c}
m+d \\
d-i
\end{array}\right)-c(-1)^{d-1} \delta(i, 1)+c(-1)^{d-1-i}\left(\begin{array}{c}
d \\
d-1-i
\end{array}\right) \\
& +\sum_{j=1}^{d-2} \sum_{\varnothing \subseteq I \subseteq[c]} \sum_{I \subseteq J \subseteq[c]}(-1)^{|J|-|I|} x_{J} \sum_{k=0}^{i} g(k)-|I| h(k) \\
& +\sum_{j=1}^{d-1} \sum_{\varnothing \subseteq J \subseteq[c]}(-1)^{|J|} x_{J} \sum_{k=0}^{i} g(k) .
\end{aligned}
$$

The following argument works for both the $i=0$ and $i>0$ case, so we speak of both simultaneously as if they were one. Let $A$ correspond to the sum indexed by $j$ where $j$ is at most $d-2$. Likewise define $B$ to be the sum indexed by $j$ where $j$ is at most $d-1$. By Lemma 14, the terms where $|J| \geqslant 2$ in $A$ will cancel all terms where $|J| \geqslant 2$ in $B$. What remains in $A$ are the terms where $|J|=1$, that is, the terms where $J=I$ and $|I|=1$. There are $c$ such terms, each contributing $\left(\begin{array}{l}d \\ j\end{array}\right)$, as the members of $\mathcal{C H}$ have cardinality $d$. For $B$, when $j \leqslant d-2$, the only terms that remain are those where $|J|$ equals 0 or 1 . This gives $c+1$ terms: one contributing $\left(\begin{array}{c}m+d \\ j\end{array}\right)$, and $c$ terms contributing $-\left(\begin{array}{l}d \\ j\end{array}\right)$. Combining this with our identity for $\Phi_{d-1}(\varnothing)$ given above, we get the following simplification.

$i=0$ :

$$
\begin{aligned}
& (-1)^{d}\left(\begin{array}{c}
m+d-1 \\
d-1
\end{array}\right)-c(-1)^{d}+c(-1)^{d-1}\left(\begin{array}{l}
d-1 \\
d-2
\end{array}\right) \\
& +c \sum_{j=1}^{d-2}\left(\begin{array}{l}
d \\
j
\end{array}\right)(-1)^{j}(\operatorname{skyt}(m+1,0, d-j+1)-\overline{\operatorname{skyt}}(0, d-j+1)) \\
& +\sum_{j=1}^{d-1}\left(\left(\begin{array}{c}
m+d \\
j
\end{array}\right)-c\left(\begin{array}{c}
d \\
j
\end{array}\right)\right)(-1)^{j} \operatorname{skyt}(m+1,0, d-j+1) .
\end{aligned}
$$


$i>0$ :

$$
\begin{aligned}
& (-1)^{d-i}\left(\begin{array}{c}
m+d \\
d-i
\end{array}\right)-c(-1)^{d-1} \delta(i, 1)+c(-1)^{d-1-i}\left(\begin{array}{c}
d \\
d-1-i
\end{array}\right) \\
& +c \sum_{j=1}^{d-2}\left(\begin{array}{l}
d \\
j
\end{array}\right) \sum_{k=0}^{i}(-1)^{j-i+k}\left(\begin{array}{c}
j \\
j-i+k
\end{array}\right)(\operatorname{skyt}(m+1, k, d-j-2 k+1) \\
& -\overline{\operatorname{skyt}}(k, d-j-2 k+1)) \\
& +\sum_{j=1}^{d-1}\left(\begin{array}{c}
m+d \\
j
\end{array}\right) \sum_{k=0}^{i}(-1)^{j-i+k}\left(\begin{array}{c}
j \\
j-i+k
\end{array}\right) \operatorname{skyt}(m+1, k, d-j-2 k+1) \\
& -\sum_{j=1}^{d-1} c\left(\begin{array}{l}
d \\
j
\end{array}\right) \sum_{k=0}^{i}(-1)^{j-i+k}\left(\begin{array}{c}
j \\
j-i+k
\end{array}\right) \operatorname{skyt}(m+1, k, d-j-2 k+1) .
\end{aligned}
$$

We now point out that remarkably, this formula no longer depends on the structure of $\mathcal{C H}$, only the cardinality. Hence, the proof proceeds as in the case of Theorem 11 in $[21]$.

\section{Bounds on $|\mathcal{C H}|$}

Our proof for the non-negativity of Theorem 1 will be purely computational. Hence, since $|\mathcal{C H}|$ is a part of our formula, having bounds on this value will be useful. We will give two particularly important bounds.

The first bound is given as follows.

Theorem 15. We have

$$
|\mathcal{C H}| \leqslant \frac{1}{m+1}\left(\begin{array}{c}
m+d \\
d
\end{array}\right)
$$

This can be recovered in multiple settings. One can find an outline of a matroid theory argument in [3, Lemma 2.7]. However, this bound also happens to be a standard coding theory result. Recall that for $S_{m, d}(\mathcal{C H})$, the circuit-hyperplanes $\mathcal{C H}$ is a subset of elements in $\left(\begin{array}{c}{[m+d]} \\ d\end{array}\right)$ so that any pair has symmetric difference at least 4 . One could equivalently describe such a set as a binary constant-weight code with hamming distance 4. In this context, the bound in Theorem 15 gives a bound on the size of a code with these conditions, as shown in [1, Theorem 12]. In fact, [1] proves a more arbitrary bound accounting for any lower bound on symmetric difference, not just 4. It is also worth noting that the proofs for this bound given in both [1] and [3] are in fact different, even when both are in the language of matroid theory. It is also worth noting one can strengthen this bound further as in the following Corollary, though it will not be used in this paper.

Corollary 16. We have

$$
|\mathcal{C H}| \leqslant \min \left\{\frac{1}{m+1}, \frac{1}{d+1}\right\}\left(\begin{array}{c}
m+d \\
d
\end{array}\right) .
$$


Proof. Recall that if $M$ is sparse paving, then so is its dual $M^{*}$. The circuit-hyperplanes of a sparse paving matroid $M$ are in bijection with the circuit-hyperplanes of $M^{*}$ (by simply taking the complement of each circuit-hyperplane with respect to the ground set). Also note that $M^{*}$ is a matroid of rank $m$ on $m+d$ elements, so the number of circuithyperplanes in $M^{*}$ (and hence also in $M$ ) is bounded by

$$
\frac{1}{d+1}\left(\begin{array}{c}
m+d \\
d
\end{array}\right)
$$

While the bound in Theorem 15 will serve useful, there will be times where it is too restrictive for our purposes. Hence, we give the following alternative bound.

Theorem 17. We have

$$
|\mathcal{C H}| \leqslant \frac{2}{m+d+2}\left(\begin{array}{c}
m+d \\
d
\end{array}\right) .
$$

Proof. Note that we have the following two chains of equivalent statements:

- $\frac{1}{m+1} \leqslant \frac{1}{d+1}$ if and only if $d \leqslant m$ if and only if $\frac{1}{m+1} \leqslant \frac{2}{m+d+2}$; and

- $\frac{1}{d+1} \leqslant \frac{1}{m+1}$ if and only if $m \leqslant d$ if and only if $\frac{1}{d+1} \leqslant \frac{2}{m+d+2}$.

Combining both of these with Corollary 16, we get

$$
|\mathcal{C H}| \leqslant \min \left\{\frac{1}{m+1}, \frac{1}{d+1}\right\}\left(\begin{array}{c}
m+d \\
d
\end{array}\right) \leqslant \frac{2}{m+d+2}\left(\begin{array}{c}
m+d \\
d
\end{array}\right)
$$

as desired.

Remark 18. Excitingly, when $m=d$, not only do the two bounds given for $|\mathcal{C H}|$ agree, but they equal the $m$ th Catalan number $C_{m}$, where

$$
C_{m}=\frac{1}{m+1}\left(\begin{array}{c}
2 m \\
m
\end{array}\right) \text {. }
$$

\section{Non-Negativity for Sparse Paving Matroids}

With the formula for Theorem 1 proven, we now move to showing that this formula is always non-negative. When $\mathcal{C H}$ is a disjoint family, this formula has a manifestly positive interpretation, as stated in the introduction of this paper. More details can be found in [21]. Otherwise, for more general cases of sparse paving matroids, there still is no manifestly non-negative expression in terms of tableaux. Instead, we show directly that our formula from Theorem 1 is non-negative by relying on the bounds given in section 5 for $|\mathcal{C H}|$, our formulas for $\operatorname{skyt}(a, i, b)$ and $\overline{\operatorname{skyt}}(i, b)$ given in section 2, and some standard algebra and calculus tools. The details for this proof will be rather technical, and our proof will need a few cases, so the proof serves more as an outline, leaving most of the work to separate Lemmas and Propositions. Throughout the proofs of this section, we use the falling factorial $(x)_{(n)}:=x(x-1) \cdots(x-n+1)$. We will also regularly use the fact $\operatorname{deg} P_{M}(t)<\frac{1}{2} \mathrm{rk} M$. That is, if $d$ is the rank of a matroid $M$, and $i$ is the power of some term in the Kazhdan-Lusztig polynomial $P_{M}(t)$, then we must have $i<d / 2$. 
Theorem 19. Let $S_{m, d}(\mathcal{C H})$ be a sparse paving matroid. Then

$$
\operatorname{skyt}(m+1, i, d-2 i+1)-|\mathcal{C H}| \cdot \overline{\operatorname{skyt}}(i, d-2 i+1) \geqslant 0 .
$$

Proof. We are able to take care of most of the cases simultaneously. For convenience, using our notation from Theorem 12, let

$$
c_{m, d}^{i}(\mathcal{C H})=\operatorname{skyt}(m+1, i, d-2 i+1)-|\mathcal{C H}| \cdot \overline{\operatorname{skyt}}(i, d-2 i+1) .
$$

Recall that $|\mathcal{C H}| \leqslant \frac{2}{m+d+2}\left(\begin{array}{c}m+d \\ d\end{array}\right)$ by Theorem 17 . Hence,

$$
c_{m, d}^{i}(\mathcal{C H}) \geqslant \operatorname{skyt}(m+1, i, d-2 i+1)-\frac{2}{m+d+2}\left(\begin{array}{c}
m+d \\
d
\end{array}\right) \cdot \overline{\operatorname{skyt}}(i, d-2 i+1) .
$$

Then by Lemma 21, this expression is non-negative for $i \geqslant 3, m \geqslant 3$, and for all possible $d$, and hence for $d>2 i$.

We now have a small number of specific cases to address. We first note that the cases for $m=0$ and $i=0$ are taken care of by Remark 13 .

When $m=1$, notice that any pair of basis elements have symmetric difference 2 , and so $|\mathcal{C H}| \leqslant 1$. In this case our desired result is immediate since by definition, we may view $\overline{\operatorname{Skyt}}(i, d-2 i+1)$ as a subset of $\operatorname{Skyt}(2, i, d-2 i+1)$.

When $m=2$, it is necessary to find a better bound on the size of $\mathcal{C H}$. It is not too much work to show that $|\mathcal{C H}| \leqslant \frac{d+2}{2}$ by using the symmetric difference condition on $\mathcal{C H}$. It is easier to work with the complements of the elements in $\mathcal{C H}$, which are elements of $\left(\begin{array}{c}{[d+2]} \\ 2\end{array}\right)$. Then it is equivalent in this case to count the size of the largest disjoint family in $\left(\begin{array}{c}{[d+2]} \\ 2\end{array}\right)$. So in the case of $m=2$ we have

$$
c_{m, d}^{i}(\mathcal{C H}) \geqslant \operatorname{skyt}(m+1, i, d-2 i+1)-\frac{d+2}{2} \cdot \overline{\operatorname{skyt}}(i, d-2 i+1),
$$

and so to prove our desired result in this case we need only to prove

$$
\operatorname{skyt}(m+1, i, d-2 i+1)-\frac{d+2}{2} \cdot \overline{\operatorname{skyt}}(i, d-2 i+1) \geqslant 0 .
$$

We do this for $i \geqslant 1$, leaving the details to Lemma 22 .

Now we move on to the remaining values of $i$, noting we need only to show them for $m \geqslant 3$. When $i=1$, one can get the following closed formula for $\operatorname{skyt}(m+1, i, d-2 i+1)$. We get

$$
\operatorname{skyt}(m+1,1, d-1)=\left(\begin{array}{c}
m+d \\
d-1
\end{array}\right)-m-d
$$

by Proposition 23. Also, note that $\overline{\operatorname{skyt}}(1, d-1)=d-1$, which can be seen by using Lemma 7 , or by simply observing that only numbers in $\{2,3,4, \ldots, d\}$ may appear below the position containing 1 in $\overline{\operatorname{skyt}}(1, d-1)$. It is also important to note that when $i=1$, 
$d \geqslant 3$. Then to get our desired result in this case, we can combine Theorem 12 and Theorem 15 and instead show that

$$
\left(\begin{array}{c}
m+d \\
d-1
\end{array}\right)-m-d-\frac{1}{m+1}\left(\begin{array}{c}
m+d \\
d
\end{array}\right)(d-1) \geqslant 0 .
$$

Lemma 24 is able to show this for $d \geqslant 3$ when $m \geqslant 4$, but only for $d \geqslant 4$ when $m=3$. This leaves the case when $m=3$ and $d=3$ to be done explicitly. Note that

$$
\operatorname{skyt}(4,1,2)=9
$$

and

$$
\overline{\operatorname{skyt}}(1,2)=2,
$$

which can be easily verified by any of our formulas from section 2 , or by hand. Then nonnegativity follows from the fact that in the special case of $m=d=3$, we can guarantee $|\mathcal{C H}| \leqslant 4$, which one verify via a constructive argument.

When $i=2$, we can use a similar strategy that we used for the $i \geqslant 3$ and $m \geqslant 3$ case described in Lemma 21. However, there will be a bit more involved here, and so we leave the details of this final case to Lemma 25.

Remark 20. In the case of $m=d=3$, it is worth noting that finding the bound $|\mathcal{C H}| \leqslant 4$ is necessary. Both bounds for $|\mathcal{C H}|$ given by Theorem 15 or Theorem 17 give $|\mathcal{C H}| \leqslant 5$, and $9-5 \cdot 2=-1$. So in this special case, we need to get a better bound on $|\mathcal{C H}|$ than what either of our two bounds could provide.

Lemma 21. Let $i$ and $m$ both be at least 3. Then

$$
\operatorname{skyt}(m+1, i, d-2 i+1)-\frac{2}{m+d+2}\left(\begin{array}{c}
m+d \\
d
\end{array}\right) \overline{\operatorname{skyt}}(i, d-2 i+1) \geqslant 0 .
$$

Proof. One can rewrite the sum in Lemma 5 using Remark 6. After doing this, letting $a=m+1$ and $b=d-2 i+1$, the $k=0$ term in the formula for $\operatorname{skyt}(m+1, i, d-2 i+1)$ is

$$
\begin{aligned}
A: & =\left(\begin{array}{c}
m+i-1 \\
i
\end{array}\right)\left(\begin{array}{c}
m+d \\
d-i
\end{array}\right) \frac{(d-i-2)_{(d-2 i-1)}(m+d-i)_{(d-2 i-1)}}{(d-2 i-1) !(m+d-i)_{(d-2 i)}} \\
& =\frac{(m+d) !(d-i-2)_{(d-2 i-1)}}{i !(m-1) !(d-i) !(m+i)(d-2 i-1) !(m+i+1)} .
\end{aligned}
$$

Utilizing Lemma 7, we have

$$
\begin{aligned}
B: & =\frac{2}{m+d+2}\left(\begin{array}{c}
m+d \\
d
\end{array}\right) \overline{\operatorname{skyt}}(i, d-2 i+1) \\
& =\frac{4(m+d) !}{m !(m+d+2)(i+1) !(i-1) !(d-2 i-1) !(d-i+1)(d-i-1)} .
\end{aligned}
$$


Note that

$$
\operatorname{skyt}(m+1, i, d-2 i+1)-\frac{2}{m+d+2}\left(\begin{array}{c}
m+d \\
d
\end{array}\right) \overline{\operatorname{skyt}}(i, d-2 i+1) \geqslant A-B,
$$

so it suffices to show $A-B \geqslant 0$. Recall that $i<d / 2$. Put another way, this says that $d-i>i>i-1$. Hence, we may combine $A-B$ in the following way.

$$
A-B=\frac{(m+d) !(m+i-1) ! p(m, i, d)}{m !(m+d+2)(i+1) !(d-i) !(m+i+1) !(d-i+1)(d-i-1)(d-2 i-1) !}
$$

where

$$
\begin{aligned}
p(m, i, d) & =(d-i-2)_{(d-2 i-1)} m(i+1)(m+d+2)(d-i+1)(d-i-1) \\
& -4(d-i)_{(d-2 i+1)}(m+i)(m+i+1) .
\end{aligned}
$$

Hence, it suffices to show that $p(m, i, d) \geqslant 0$. We can, in fact, reduce the problem further by simplifying $p(m, i, d)$. Observe that

$p(m, i, d)=(d-i-1)_{d-2 i}[m(i+1)(m+d+2)(d-i+1)-4(m+i)(m+i+1)(d-i)]$,

so it now suffices to show

$$
q(m, i, d):=m(i+1)(m+d+2)(d-i+1)-4(m+i)(m+i+1)(d-i) \geqslant 0 .
$$

We show this for $m, i \geqslant 3$ by viewing $q$ as a function of $m$. The desired result follows from the following three claims for $q$ as a function of $m$.

1. $q$ is quadratic and concave up;

2. the critical point of $q$ is negative; and

3. $q(m, i, d) \geqslant 0$ for $m=3$.

Showing these are elementary exercises in algebra and calculus, so we just highlight the important parts.

For claim (1), note that the coefficient of $m^{2}$ in $q(m, i, d)$ is $(i+1)(d-i+1)-4(d-i)$, and that we assume $d>2 i$ and $i \geqslant 3$. Hence this coefficient is non-negative.

For claim (2), it suffices to show the coefficient of $m$ in $q(m, i, d)$ is positive. This coefficient is

$$
(i+1)(d+2)(d-i+1)-4(i+1)(d-i)-4 i(d-i) .
$$

Using the fact that $d>2 i$, one can show this is an increasing function in $d$ and is nonnegative when $d=2 i$.

For claim (3), it suffices to show $q(3, i, d)$ is an increasing function in $d$ and that $q(3, i, 2 i)$ is non-negative. This works out similarly to claim (2). 
Lemma 22. Let $i \geqslant 1$ and $m=2$. Then

$$
\operatorname{skyt}(m+1, i, d-2 i+1)-\frac{d+2}{2} \overline{\operatorname{skyt}}(i, d-2 i+1) \geqslant 0 .
$$

Proof. As in Lemma 21, keeping in mind that $m=2$, set

$$
A:=\frac{(d+2) !(d-i-2)_{(d-2 i-1)}}{i !(d-i) !(i+2)(d-2 i-1) !(i+3)}
$$

and

$$
\begin{aligned}
B: & =\frac{d+2}{2} \overline{\operatorname{skyt}}(i, d-2 i+1) \\
& =\frac{d !(d+2)}{(i+1) !(i-1) !(d-2 i-1) !(d-i+1)(d-i-1)} .
\end{aligned}
$$

It follows from the proof of Lemma 21 that $\operatorname{skyt}(m+1, i, d-2 i+1) \geqslant A$ for $m=2$, and so the desired result follows if we show $A-B \geqslant 0$. Observe that

$$
A-B=\frac{d !(d+2) p(i, d)}{(i+3) !(d-i) !(d-2 i-1) !(d-i+1)(d-i-1)}
$$

where

$p(i, d):=(d-i-2)_{(d-2 i-1)}(d+1)(i+1)(d-i+1)(d-i-1)-(i+2)(i+3)(d-i)_{(d-2 i+1)}$.

Hence, it suffices to show that $p(i, d)$ is non-negative. One can factor $p(i, d)$ to reduce the problem further:

$$
p(i, d)=(d-i-1)_{(d-2 i)}[(d+1)(i+1)(d-i+1)-(i+2)(i+3)(d-i)],
$$

and so it suffices to show that

$$
q(i, d):=(d+1)(i+1)(d-i+1)-(i+2)(i+3)(d-i)
$$

is non-negative. Since in the context of Kazhdan-Lusztig polynomials we have $d>2 i$, we may set $d=2 i+j$ for $j \geqslant 1$. Then $q(i, 2 i+j)$ is quadratic in $j$ and we have the following values of $\left[j^{\ell}\right] q(i, 2 i+j)$ :

$$
\begin{aligned}
& {\left[j^{2}\right] q(i, 2 i+j)=i+1,} \\
& {\left[j^{1}\right] q(i, 2 i+j)=2 i^{2}-4,}
\end{aligned}
$$

Remaining terms: $i^{3}-2 i+1$.

When $i \geqslant 2$, all three values are individually positive. If $i=1$, then

$$
q(1, j+2)=2 j^{2}-2 j
$$

which is non-negative for all $j \geqslant 1$, giving our desired result. 


\section{Proposition 23.}

$$
\operatorname{skyt}(m+1,1, d-1)=\left(\begin{array}{c}
m+d \\
d-1
\end{array}\right)-m-d
$$

Proof. Note that if $\alpha \in \operatorname{Skyt}(m+1,1, d-1)$, it is made up of two "tails", one of length $m+1$ extending down, and the other of length $d-1$ extending up, so that the two tails overlap in exactly two positions. See the below figure for a schematic of $\alpha$, with some entries labeled.

$$
m+1\left\{\begin{array}{l|l}
\frac{\square}{w} & y \\
\hline x & z \\
\hline & \\
\hline &
\end{array}\right] d-1
$$

Note that there are $m+d$ positions in this tableau, and we require that $w<y$ and $x<z$. Now, pick an element $S \in\left(\begin{array}{c}{[m+d]} \\ d-1\end{array}\right)$. The number of elements of $\operatorname{Skyt}(m+$ $1,1, d-1)$ is equivalent to the number of $S$ that appear as the right tail in an element in $\operatorname{Skyt}(m+1,1, d-1)$, as the entries of one tail determine the entries of the other. It is easiest to count the complement, that is, the $S$ that will not appear as the the right tail in an element of $\operatorname{Skyt}(m+1,1, d-1)$. These are the $S$ that force $w>y, x>z$, or both. We leave it to the reader to verify that the complement has size $m+d$.

Lemma 24. We have

$$
\left(\begin{array}{c}
m+d \\
d-1
\end{array}\right)-m-d-\frac{1}{m+1}\left(\begin{array}{c}
m+d \\
d
\end{array}\right)(d-1) \geqslant 0
$$

for $d \geqslant 3$ when $m \geqslant 4$, and $d \geqslant 4$ when $m=3$.

Proof. We start by rewriting of our expression of interest.:

$$
\left(\begin{array}{c}
m+d \\
d-1
\end{array}\right)-m-d-\frac{1}{m+1}\left(\begin{array}{c}
m+d \\
d
\end{array}\right)(d-1)=(m+d)\left(\frac{(m+d-1)_{(d-2)}}{d !}-1\right) .
$$

Hence, if

$$
f(m, d):=\frac{(m+d-1)_{(d-2)}}{d !}
$$

it suffices to show $f(m, d) \geqslant 1$. As a function in $m, f(m, d)$ is increasing. Also,

$$
f(4, d)=\frac{(d+3)_{(d-2)}}{d !}=\frac{(d+3) !}{5 ! d !}=\frac{1}{20}\left(\begin{array}{c}
d+3 \\
3
\end{array}\right) .
$$


See that $f(4, d)$ is increasing in $d$ and also $f(4,3)=1$. So when $m \geqslant 4$, we have our desired result for $d \geqslant 3$. When $m=3$, we observe that

$$
f(3, d)=\frac{(d+2)_{(d-2)}}{d !}=\frac{(d+2) !}{4 ! d !}=\frac{1}{12}\left(\begin{array}{c}
d+2 \\
2
\end{array}\right) .
$$

Note that $f(3, d)$ is increasing in $d$, and $f(3,4)=\frac{15}{12}$.

Lemma 25. If $m \geqslant 3$, we have

$$
c_{m, d}^{2}(\mathcal{C H}) \geqslant 0 .
$$

Proof. It will be important to remember that since $i=2$, we have $d \geqslant 5$ by the degree requirement on Kazhdan-Lusztig polynomials.

To show our desired result, we will need two separate cases. First suppose $m \geqslant d$. Note then we already have $m \geqslant 3$ since $d \geqslant 5$. As in Lemma 21, accounting for the fact that in this case $i=2$, let

$$
\begin{aligned}
A: & =\frac{(m+d) !(d-4)_{(d-5)}}{2(m-1) !(d-2) !(m+2)(d-5) !(m+3)} \\
& =\frac{(m+d) !(d-4) m(m+1)}{2(m+3) !(d-2) !} .
\end{aligned}
$$

Also similarly to Lemma 21, but using the bound from Theorem 15 for $|\mathcal{C H}|$, let

$$
\begin{aligned}
B: & =\frac{1}{m+1}\left(\begin{array}{c}
m+d \\
d
\end{array}\right) \frac{2 \cdot d !}{6(d-5) !(d-1)(d-3)} \\
& =\frac{(m+d) !(d-2)(d-4)}{3(m+1) !(d-1) !} .
\end{aligned}
$$

A combination of Theorem 12, Theorem 15, and the proof of Lemma 21 implies that

$$
c_{m, d}^{2}(\mathcal{C H}) \geqslant A-B,
$$

and so it remains to show that $A-B \geqslant 0$ when $m \geqslant d$. Notice that

$$
A-B=\frac{(m+d) !(d-4) f(m, d)}{6(m+3) !(d-1) !}
$$

where

$$
f(m, d):=3 m(m+1)(d-1)-2(d-2)(m+2)(m+3) .
$$

Hence, it suffices to show that $f(m, d) \geqslant 0$. Since $m \geqslant d$, we set $m=d+j$, for $j \geqslant 0$. Then $f(d+j, d)$ is quadratic in $j$ and we have

$$
\begin{aligned}
{\left[j^{2}\right] f(d+j, d) } & =d+1, \\
{[j] f(d+j, d) } & =2 d^{2}-5 d+17, \text { and }
\end{aligned}
$$

Remaining terms of $f(d+j, d): d^{3}-6 d^{2}+5 d+24$. 
One can verify each of these are positive when $d=5$, and that each is increasing in $d$ when $d \geqslant 5$. Hence, this shows that $c_{m, d}^{2}(\mathcal{C H}) \geqslant 0$ so long as $m \geqslant d$.

Now we show the same result holds when $d \geqslant m$. To do this, we reuse $A$ as above, and redefine $B$ using our bound from Theorem 17 :

$$
\begin{aligned}
B: & =\frac{2}{m+d+2}\left(\begin{array}{c}
m+d \\
d
\end{array}\right) \frac{2 \cdot d !}{6(d-5) !(d-1)(d-3)} \\
& =\frac{2(m+d) !(d-2)(d-4)}{3(m+d+2) m !(d-1) !} .
\end{aligned}
$$

For similar reasons as before, $c_{m, d}^{2}(\mathcal{C H}) \geqslant 0$ if $A-B \geqslant 0$. Note that

$$
A-B=\frac{(m+d) !(d-4)(m+1) g(m, d)}{6(m+3) !(d-1) !}
$$

where

$$
g(m, d):=3 m(m+d+2)(d-1)-4(d-2)(m+2)(m+3) .
$$

Observe that $g$ is a concave up quadratic function in $d$. If one expands the function, its vertex can be seen to occur at

$$
d=\frac{m^{2}+17 m+24}{6 m} .
$$

However, note that this value is less than $m$ so long as $m \geqslant 5$ since

$$
\frac{m^{2}+17 m+24}{6 m} \leqslant m \text { if and only if }-5 m^{2}+17 m+24 \leqslant 0 .
$$

Hence, this says that $g(m, d)$ is increasing in $d$ when $d \geqslant m \geqslant 5$. Also, when $m=3$ the vertex for $g$ is at approximately $d=4.67$ and when $m=4$ the vertex for $g$ is at $d=4.5$. We know that $d \geqslant 5$ regardless of its relation to $m$, so we have in fact shown that $g$ is increasing in $d$ for any $m \geqslant 3$ when $d \geqslant m$. Moreover, one can verify

$$
g(m, m)=2\left(m^{3}-6 m^{2}+5 m+24\right) \geqslant 0
$$

so long as $m \geqslant 5$. Also, note that $g(3,5)=0$ and $g(4,5)=24$. Hence $g(m, d)$ is always non-negative for $d \geqslant m$ when $m \geqslant 3$.

\section{$7 \quad$ Integral Identities}

The following proposition is due to [16, Identity 2.110.8], though it is not immediately obvious until one makes the appropriate substitutions and simplifications.

Proposition 26. Let $a, b$ be positive integers. Then

$$
\int y^{a}(1-x y)^{b} d y=a ! b ! \sum_{k=0}^{b} \frac{(1-x y)^{b-k} y^{a+k+1} x^{k}}{(a+k+1) !(b-k) !} .
$$


Corollary 27. Let $a, b$ be positive integers. Then

$$
\int_{0}^{1} y^{a}(1-x y)^{b} d y=a ! b ! \sum_{k=0}^{b} \frac{(1-x)^{b-k} x^{k}}{(a+k+1) !(b-k) !} .
$$

Corollary 28. Let $a, b$ be positive integers. Then

$$
\int_{0}^{y} x^{a}(1-x)^{b} d x=a ! b ! \sum_{k=0}^{b} \frac{(1-y)^{b-k} y^{a+k+1}}{(a+k+1) !(b-k) !} .
$$

Proposition 29. Let $x_{0}, x_{1}, \ldots, x_{i}$ be a list of $i+1$ variables. Set $h_{1}\left(x_{1}\right)=\int_{0}^{x_{1}} x_{0}^{a}(1-$ $\left.x_{0}\right)^{b} d x_{0}$, and for $i>1$ define $h_{i}\left(x_{i}\right)=\int_{0}^{x_{i}} h_{i-1}\left(x_{i-1}\right) d x_{i-1}$. Then

$$
\int_{0}^{1} h_{i}\left(x_{i}\right) d x_{i}=\frac{a !(b+i) !}{i !(a+b+i+1) !} .
$$

Proof. Using Corollary $28 i$ times, we get the following expression for $h_{i}\left(x_{i}\right)$.

$$
h_{i}\left(x_{i}\right)=a ! b ! \sum_{k_{1}=0}^{b} \sum_{k_{2}=0}^{b-k_{1}} \sum_{k_{3}=0}^{b-k_{1}-k_{2}} \cdots \sum_{k_{i}=0}^{b-\sigma} \frac{x_{i}^{a+\sigma+k_{i}+i}\left(1-x_{i}\right)^{b-\sigma-k_{i}}}{\left(a+\sigma+k_{i}+i\right) !\left(b-\sigma-k_{i}\right) !},
$$

where $\sigma=k_{1}+k_{2}+\cdots+k_{i-1}$. Noting that

$$
\int_{0}^{1} x_{i}^{a+\sigma+k_{i}+i}\left(1-x_{i}\right)^{b-\sigma-k_{i}} d x_{i}=\frac{\left(a+\sigma+k_{i}+i\right) !\left(b-\sigma-k_{i}\right) !}{(a+b+i+1) !},
$$

we may use (5) to write

$$
\begin{aligned}
& \int_{0}^{1} h_{i}\left(x_{i}\right) d x_{i} \\
& =a ! b ! \sum_{k_{1}=0}^{b} \sum_{k_{2}=0}^{b-k_{1}} \sum_{k_{3}=0}^{b-k_{1}-k_{2}} \cdots \sum_{k_{i}=0}^{b-\sigma} \frac{\left(a+\sigma+k_{i}+i\right) !\left(b-\sigma-k_{i}\right) !}{\left(a+\sigma+k_{i}+i\right) !\left(b-\sigma-k_{i}\right) !(a+b+i+1) !} \\
& =\frac{a ! b !}{(a+b+i+1) !} \sum_{k_{1}=0}^{b} \sum_{k_{2}=0}^{b-k_{1}} \sum_{k_{3}=0}^{b-k_{1}-k_{2}} \cdots \sum_{k_{i}=0}^{b-\sigma} 1,
\end{aligned}
$$

which simplifies using Proposition 30 to

$$
\frac{a ! b !}{(a+b+i+1) !}\left(\begin{array}{c}
b+i \\
i
\end{array}\right)=\frac{a !(b+i) !}{i !(a+b+i+1) !} .
$$




\section{Proposition 30.}

$$
\sum_{k_{1}=0}^{b} \sum_{k_{2}=0}^{b-k_{1}} \sum_{k_{3}=0}^{b-k_{1}-k_{2}} \cdots \sum_{k_{i}=0}^{b-\sigma} 1=\left(\begin{array}{c}
b+i \\
i
\end{array}\right)
$$

where $\sigma=k_{1}+k_{2}+\cdots+k_{i-1}$.

Proof. It is helpful to first reindex the summations so that they start at 1 instead of 0 . Then the identity holds from counting the below set in two ways.

$$
\bigcup_{x_{1} \in[b+1]} \bigcup_{x_{2} \in[b+2] \backslash\left[x_{1}\right]} \bigcup_{x_{3} \in[b+3] \backslash\left[x_{2}\right]} \ldots \bigcup_{x_{i} \in[b+i] \backslash\left[x_{i-1}\right]}\left\{x_{1}, x_{2}, \ldots, x_{i}\right\} .
$$

\section{Acknowledgments}

The authors would like to thank Nicholas Proudfoot and Jacob Matherne for their helpful comments and feedback. We also thank the anonymous referees for feedback and corrections that helped improve the quality of this paper.

\section{References}

[1] E. Agrell, A. Vardy, K. Zeger, Upper bounds for constant-weight codes. (English summary) IEEE Trans. Inform. Theory 46 (2000), no. 7, 2373-2395.

[2] A. Björner, F. Brenti, Combinatorics of Coxeter groups. Graduate Texts in Mathematics, vol. 231, Springer, New York, 2005.

[3] J. Bonin, Basis-exchange properties of sparse paving matroids. Adv. in Appl. Math. 50 (2013), no. 1, 6-15.

[4] T. Braden, J. Huh, J. P. Matherne, N. Proudfoot, B. Wang, A Semi-Small Decomposition of the Chow Ring of a Matroid. arXiv:2002.03341.

[5] T. Braden, J. Huh, J. P. Matherne, N. Proudfoot, B. Wang, Singular Hodge Theory for Combinatorial Geometries. arXiv:2010.06088.

[6] T. Braden, A. Vysogortes, Kazhdan-Lusztig Polynomials of Matroids Under Deletion. (English summary) Electron. J. Combin. 27 (2020), no. 1, \#P1.17.

[7] F. Brenti, Twisted incidence algebras and Kazhdan-Lusztig-Stanley functions. Adv. Math. 1999, 148(1): 44-74.

[8] F. Brenti, P-kernels, IC bases and Kazhdan-Lusztig polynomials. J. Algebra, 2003, 259(2): 613-627.

[9] H. H. Crapo and G-C. Rota, On the foundations of combinatorial theory: Combinatorial geometries. The M.I.T. Press, Cambridge, Mass.-London, preliminary edition, 1970.

[10] B. Elias, N. Proudfoot, M. Wakefield, The Kazhdan-Lusztig polynomial of a matroid. Adv. Math. 299 (2016), 36-70. 
[11] B. Elias, G. Williamson, The Hodge theory of Soergel bimodules. (English summary) Ann. of Math. (2) 180 (2014), no. 3, 1089-1136.

[12] L. Ferroni, L. Vecchi, Matroid Relaxations and Kazhdan-Lusztig non-Degeneracy. arXiv:2104.14531

[13] A. L.L. Gao, M. H.Y. Xie, A. L.B. Yang, The equivariant inverse Kazhdan-Lusztig polynomials of uniform matroids. arXiv:2105.08546.

[14] K. Gedeon, Kazhdan-Lusztig polynomials of thagomizer matroids. Electron. J. Combin. 24 (2017), no. 3, \#P3.12.

[15] A. Gao, L. Lu, M. Xie, A. Yang, P. Zhang, The Kazhdan-Lusztig polynomials of uniform matroids. (English summary) Adv. in Appl. Math. 122 (2021), Paper No. 102117, 24 pp.

[16] I. S. Gradshteyn, I. M. Ryzhik, Table of integrals, series, and products. Translation edited and with a preface by Alan Jeffrey and Daniel Zwillinger. Seventh edition. Elsevier/Academic Press, Amsterdam, 2007.

[17] K. Gedeon, N. Proudfoot, B. Young, The equivariant Kazhdan-Lusztig polynomial of a matroid. J. Combin. Theory Ser. A 150 (2017), 267-294.

[18] K. Gedeon, N. Proudfoot, B. Young, Kazhdan-Lusztig polynomials of matroids: a survey of results and conjectures. (English summary) Sém. Lothar. Combin. 78B (2017), Art. 80, 12 pp.

[19] T. Karn, M. Wakefield, Stirling numbers in braid matroid Kazhdan-Lusztig polynomials. Adv. in Appl. Math. 103 (2019), 1-12.

[20] D. Kazhdan and G. Lusztig, Representations of Coxeter groups and Hecke algebras. Invent. Math. 53 (1979), no. 2, 165-184.

[21] K. Lee, G. D. Nasr, J. Radcliffe, A Combinatorial Formula for Kazhdan-Lusztig Polynomials of $\rho$-Removed Uniform Matroids. Electron. J. Combin. 27 (2020), no. 4, \#P4.7.

[22] L. Lu, M. Xie, A. Yang, Kazhdan-Lusztig polynomials of fan matroids, wheel matroids and whirl matroids. arXiv:1802.03711.

[23] D. Mayhew, M. Newman, D. Welsh, G. Whittle, On the asymptotic proportion of connected matroids, European Journal of Combinatorics, 32 (2011), no. 6, 882-890.

[24] R. Pendavingh, J. van der Pol, On the number of matroids compared to the number of sparse paving matroids, Electron. J. Combin. 22 (2015), no. 2, \#P2.51.

[25] N. Proudfoot, Equivariant Kazhdan-Lusztig polynomials of q-niform matroids. (English summary) Algebr. Comb. 2 (2019), no. 4, 613-619.

[26] N. Proudfoot, The algebraic geometry of Kazhdan-Lusztig-Stanley polynomials. EMS Surv. Math. Sci. 5 (2018), no. 1, 99-127.

[27] N. Proudfoot, M. Wakefield, B. Young, Intersection cohomology of the symmetric reciprocal plane. J. Algebraic Combin. 43 (2016), no. 1, 129-138. 
[28] P. Polo, Construction of arbitrary Kazhdan-Lusztig polynomials in symmetric groups. Represent. Theory 3 (1999), 90-104 (electronic).

[29] P. Nelson, Almost all matroids are nonrepresentable. Bull. Lond. Math. Soc. 50 (2018), no. 2, 245-248.

[30] R. P. Stanley, Polygon dissections and standard Young tableaux. J. Combin. Theory Ser. A, 76 (1996), 175-177.

[31] R. P. Stanley, Subdivisions and local h-vectors. J. Amer. Math. Soc. 5 (1992), no. 4,805-851.

[32] M. Wakefield, Partial flag incidence algebras. arXiv:1605.01685.

[33] M. Xie, P. Zhang, Equivariant Kazhdan-Lusztig polynomials of thagomizer matroids. Proc. Amer. Math. Soc. 147 (2019), no. 11, 4687-4695. 